\title{
Plataformas
}

\section{mediáticas y niveles de análisis}

\section{POR JOSÉ LUIS FERNÁNDEZ}

j_fernandez@szinfonet.com.ar Facultad de Ciencias Sociales, Universidad de Buenos Aires (UBA), Argentina.

Fecha de recepción: 20/9/2016

Fecha de aceptación: 9/10/2016

\section{RESUMEN}

En el trabajo de investigación sobre fenómenos de actualidad conviven profundas transformaciones de nuestros objetos junto con el avance de nuestros estudios sobre ellos. Por eso, cada nueva publicación exige trabajar en tensión entre diversas temporalidades y espacialidades. En este artículo presentaremos descripciones acerca de cómo algunas delas denominadas redes sociales se van convirtiendo en complejas plataformas multimedia. Esa actividad multimediática convive con prácticas particulares de interacción de la vida mediatizada, algunas muy novedosas, pero otras con una larga tradición.

Aquí se propondrá que, para comprender el funcionamiento de ciertos aspectos del intercambio en plataformas, se deben investigar, al menos, dos niveles diferentes: por un lado, entender cómo las plataformas se diferencian mientras interactúan entre sí; por el otro, con un enfoque sociosemiótico, cómo su vida mediática se constituye con micro sistemas de intercambio discursivo. Creemos que la tensión entre ese campo en intersección entre las plataformas y las acciones discursivas micro permitirá comprender cómo es que vemos el estado actual de las todavía denominadas nuevas mediatizaciones.

PALABRAS CLAVE: plataformas, mediatizaciones, interacciones, intercambios discursivos.

\begin{abstract}
At work research on present process coexists deep transformations of our objects with the progress of our studies on them. Therefore, each new publication requires work in tension between various temporalities and spatialities. In this article we will present descriptions about how some of social media and networks social are being transforming in complex multimedia platforms. In the same time, that multi-media activity coexists with partic-
\end{abstract}


ular practices of interaction of the mediatizated life, some pf them very innovative, but others coming on from a long tradition.

To understand the functioning of those multi-media platforms, two different levels should be investigated: on the one hand, understand how platforms are different proposals to interact among themselves; on the other hand, with a sociosemiotics approach, how micro media discursive systems coexists in each platform. We believe that to focus on the media crossroads between media platforms and micro discursive action will allow to understand the current status of the mediazations still called new media.

KEYWORDS: Platforms, mediations, interactions, discursive exchanges.

\section{INTRODUCCIÓN}

Este artículo trata de explorar la experiencia de la denominada "socialidad por plataformas" (Van Dijck1, 2016, p. 19), que excede a la socialidad entendida como contactos e interacciones entre individuos y/o grupos, para recuperar modos de intercambio discursivo de las mediatizaciones previas, aunque no "viejas". Esa recuperación no implica cuestionar el crecimiento de los intercambios en networking, pero sí introducirnos un paso más en lo que hemos denominado como postbroadcasting: un espacio central en el ecosistema de las mediatizaciones sociales en el que están en tensión intercambios networking con intercambios broadcasting.

Sibien este artículo tendrá un enfoque teórico y metodológico, se trata de un paso más en nuestro proyecto de investigación en el que venimos estudiando, entre otros fenómenos, plataformas mediáticas que de alguna manera incluyen o cuestionan procesos de construcción de espacios de interacción e intercambio propuestos por esas plataformas ${ }^{2}$. Hemos publicado sucesivos trabajos sobre una plataforma multimediática, Vorterix.com (Fernández, 2016), y en este momento estamos estableciendo sus parecidos y diferencias con otras plataformas.

Por otra parte, cada artículo que publicamos relacionado con nuestros trabajos de investigación nos obliga a una especie de recorrido en espiral: debemos recuperar, y discutir previamente algo recientemente escrito, para apoyar el nuevo avance que nos proponemos presentar. En este sentido, seguimos el camino que, entre otros, ha desplegado Lisa Gitelman (2006), quien comenzaba su libro sobre los new media con el fonógrafo, que a fines del siglo XIX era un

1 Johanna Francisca Theodora María «José» Van Dijck.

2 Se trata del Proyecto de investigación, con subsidio UBACyT, que lleva por título: Letra, imagen, sonido. Convergencias y divergencias metodológicas y teóricas en el estudio de las relaciones entre los medios y el espacio urbano. 2014-2017. En ese sentido, buena parte del artículo se debe a la tarea de un grupo con el que venimos investigando cuestiones relacionadas a las mediatizaciones y sus relaciones con otras áreas de la vida social (música, gobierno, etc.). 
new media (luego precisaremos el concepto de medio de acuerdo a nuestra experiencia de investigación).

Se ha advertido que estamos en el tercer momento en la investigación de lo que se denominan nuevas mediatizaciones: en él, ya no deberíamos estar asombrados por el poderío del acceso a la información basado en lo digital y en la Internet (es más, ya sabemos que no es la única red posible), ni por las infinitas posibilidades de interacción horizontal que ofrecen las redes sociales mediáticas (sí, también hubo que admitir la importancia de las redes sociales soportadas total o parcialmente en los intercambios cara a cara).

Este tercer momento se caracteriza porque ya tenemos experiencia prolongada como usuarios de diversas herramientas en red y porque contamos con buenas experiencias de investigación en muchas de ellas. También conocemos sus límites: no todos los usuarios están interesados en interactuar y muchas prácticas que encontramos en las redes se sabe que provienen de las experiencias previas de los usuarios, más que de las propuestas de diseño y programación de los propietarios originales. La provocadora pregunta sobre "qué hace el mundo con los social media" (Miller, 2016), invirtiendo la pregunta habitual, convive y cobra fuerza frente a la más mcluhiana pero también sugerente concepción de que la sociedad es moldeada por las mediatizaciones en general, y por las redes en particular: es así en los casos en que "el software toma el mando" (Manovich, 2013)

Es decir, que transitamos un momento de redefiniciones de nuestros objetos y en el que, si bien estamos todavía lejos de entrever cómo será el cuarto momento, parece que resultará un producto de juegos de tensiones entre mediatizaciones interindividuales y mediatizaciones de alcance relativamente masivo. En cierto sentido, esas tensiones entre extremos también están anunciadas por los dos polos entre los que se mueven las investigaciones publicadas: las descripciones detalladas de lo individual-etnográfico y las búsquedas extensas de nuevas relaciones a través del big-data. Las propuestas de investigación que se presentan aquítratan degenerar espaciosintermedios dereflexión eindagación.

En términos generales, consideramos que las mediatizaciones se estudian desde perspectivas macro, conceptualizando fenómenos complejos de alcance genéricamente social o cultural y, en el otro, intercambios mediáticos micro, circunscriptos a una localización, un medio o un campo conceptual conflictivo (violencia, género, política, etc.).Entendemos que es desde el punto de vista micro donde se generan novedades; pero para articular con lo macro, hacen falta intervenciones einterpretaciones médium, intermediarias entre ambos niveles (Fernández,2012).Si de datos micro se sacan rápidamente conclusiones macro, suele ocurrir que lo macro ya sabido o intuido, se impone sobre las novedades

3 Debo el contacto con el trabajo de Miller a la Cátedra de Antropología Social, dirigida por Gerardo Halpern. Agradezco a él, a Mercedes Calzado y a Victoria Irrisarri, del equipo, el aprendizaje y la posibilidad de discutir y profundizar con ellos. 
halladas en lo micro. Pero las distintas extensiones de los objetos estudiados y sus campos conceptuales son relativos a los fenómenos que se estudia.

En este artículo trataremos de revisar, como un nivel médium de nuestro trabajo, algunosfenómenos particulares delas plataformas mediáticas, que son las actuales interfaces entre mediatizaciones y actores de la mediatización. Nos preguntaremos si algunas de esas plataformas están tendiendo a constituirse en plataformas de interfaz de inicio en los diferentes dispositivos que tienden a converger: smartphones, tablets, televisores y computadores. Aunque no lo discutamos especialmente, estamos ubicándonos allí en el campo de los ecosistemas de los medios.

Por otro lado, hacia el final del artículo, nos introduciremos en la reflexión y descripción de fenómenos micro pero particulares en la vida de las plataformas, como la recepción de celdas de broadcasting en plataformas en red, la secuencias de posteos y comments, ejemplificando con muros de Facebook y observando un fenómeno muy particular como el de los megusteos diferentes que cualquier usuario de Facebook sabe reconocer. En ese nivel micro, propondremos la necesidad de recuperar enfoques diferentes de la sociosemiótica de las mediatizaciones en el cruce de tensiones entre lo etnográfico y la sombra deseada del big-data.

\section{PLATAFORMAS MEDIÁTICAS: MÁS ALLÁ DE LAS REDES}

El término de plataformas viene siendo utilizado desde lo que hemos denominado el segundo momento de estudios sobre las nuevas mediatizaciones. Pero si bien en Jenkins (2008) como en Scolari (2008) son mencionadas como fenómenos diferenciados delos medios, ya cuando el propio Scolari (2013) trata de reconstruirla "galaxia semántica" que constituyen las narrativas transmedia, las plataformas mediáticas conviven con cross-media, hybrid media, transmedia worlds, transmedial interactions, multidmodality, etc. De todos modos es en Van Dijck $(2013 ; 2016)$ donde se registra un esfuerzo de diferenciación de las plataformas como fenómeno mediático, poniéndolas en serie con la definición de media de Gitelman, para quien son:

Social realized structures of communication, where structures include both technological forms and their associated protocoles, and where communication is a social practice, a ritualizated collocation of different people in the same mental map, sharing or engaged with popular ontologies of representation (2006, p. 24)

Como se ve, estructuras, formas tecnológicas, ritos, mapas mentales, ontologías de representación, términos relativamente abstractos de los que las plataformas serán un punto más del pasaje del cara a cara ala mediatización. Nociones de

4 Una traducción del párrafo en Van Dijck (2016, p. 20). 
ecología de los medios que se relacionan casi naturalmente, aunque sin abandonar la tensión con otros fenómenos macro de la vida social como la economía, la cultura, la política, el comercio, etc. Nos parece que este tipo de definiciones, de nivel macro, no representan bien los matices de diferenciación entre fenómenos mediáticos que, entre otros espacios, se manifiestan en las plataformas.

Es por esa advertencia sobre lo macro que cuando nos referimos a plataformas mediáticas, seguimos sólo parcialmente el trabajo clave de Van Dijck (2016), que considera a las plataformas de socialización como conjuntos de tecnologías (softwares y hardwares, interfaces explícitas o no), usos, modelos de negocio, etc.; que soportan y/o constituyen diferentes tipos de intercambio social (desde nuestro punto de vista, decimos intercambio social o discursivo social, que es el objeto específico de nuestro trabajo). Como se ve, se trata también de una definición macro.

Un punto de vista muyvalioso en el enfoque de Van Dijck aparece en el capítulo "Desmontando plataformas, reconstruyendo socialidad" (2016,p.47). Allí la investigadora holandesa relaciona el lanzamiento por Apple de iTunes con el posterior del iPod y con el desarrollo ulterior de las listas de reproducción y los modelos de negocio que llevan hoy a fenómenos como los de Spotify. Es decir, quela idea de plataforma en Van Dijckes sistémica y transmedia. En este sentido, se relaciona con lo anteriormente citado de Scolari: hay niveles de relacionamiento entre mediatizaciones de las quelas plataformas son una manifestación material, entre otras posibles. Así, desde el punto de vista delas mediatizaciones -y entendemos al nivel de las plataformas como fenómenos que hoy son una parte importante en la vida de aquellas-, el fenómeno plataforma es un nivel de intercambio mediático múltiple que obliga a revisar no sólo la definición de medio, sino también, a esta altura, las de redes o las de medios sociales.

Esto es muy importante para nosotros porque nuestras investigaciones han puesto en evidencia un uso de las mediatizaciones de sonido que constituyen una especie de plataforma de uso aplicada de modo relativamente independiente por los usuarios: el transeúnte que recorre el espacio urbano con auriculares alterna la escucha radiofónica (en broadcasting) con sus playlists musicales (en tensión entre la oferta de sitios como Spotify, Deezer, etc. y la propia selección; es decir, la oferta social y la combinatoria individual, alternando con podcasting presentes en otras plataformas y con la escucha de mensajes de audio a través de aplicaciones como WhatsApp. Es decir que el transeúnte que utiliza auriculares funciona en plataforma.

Ese modo mediatizado de recorrer el espacio urbano es muy revelador. Por un lado, muestra que esa vida mediatizada convive en su conjunto, y en competencia, con las interacciones focalizadas o no focalizadas en el otro copresente que Joseph (1999) toma de Goffman: interacciones simbólicas complejas o de puro contacto, disponibles para ser focalizadas o no. Por otro lado, representa el modo de vida en las sociedades mediatizadas que tiende a abandonar defi- 
nitivamente las formas de espectación relativamente serenas y en posiciones estables, por maneras que suponen decidir momentos y modos de recepción, combinados con modos de producción y emisión. En esos sistemas de intercambios más inestables se encuentran prácticas aproximadas al broadcasting (de ego para muchos más o menos indeterminados) o puramente en networking: entre pares, amigos o colegas.

\section{MEDIATIZACIONES Y PLATAFORMAS: UN NIVEL MÉDIUM}

Para nosotros, entonces, son plataformas de mediatización los complejos sistemas multimodality de intercambios discursivos mediatizados que permiten la interacción o, al menos, la copresencia entre diversos sistemas de intercambio (cross, inter, multi o transmedia). Creemos que es necesario, antes de avanzar en lo que nos interesa en este artículo, proponer las definiciones que venimos aplicando en nuestro trabajo para que se entienda el fundamento de nuestras propuestas.

En primer lugar, denominamos mediatización a todo sistema de intercambio discursivo de vida social que se realiza mediante la presencia de dispositivos técnicos que permiten la modalización espacial, temporal o espacio-temporal del intercambio (directo, grabado, presencia o no del cuerpo, indicialidad, iconocidad o simbolicidad, etc.). En ese sentido, una mediatización siempre se opone a los intercambios cara a cara, pero por supuesto, como en toda la vida social, encontramos la presencia de zonas grises, como las conferencias con amplificación de sonido y soporte de medios visuales.

Por otro lado, para que una mediatización sea considerada como una parte relativamente estable de la vida social y cultural debe estar constituida por al menos tres niveles defenómenos, de vida relativamente independiente entre sí:

- Los ya mencionados dispositivos técnicos que tienen una dinámica propia vinculada, por ejemplo, a momentos sociales más o menos "invencionistas".

- La presencia habitual de géneros y estilos discursivos más o menos populares o masivos (no conviene en este nivel introducir el fenómeno de los lenguajes, porque así como sabemos ahora que hay más de un lenguaje radiofónico o televisivo, también notamos que en las nuevas mediatizaciones se presentan tanto hibridaciones como esfuerzos teóricos para construir categorías metadiscursivas de base metafísica.

- De la vida de una mediatización se deben entender sus usos sociales (información, entretenimiento, educación, competencia, lucha política, interindividualidad, etc.). La comprensión de sus usos sociales debeincluirlapresencia de usos desviantes como, por ejemplo, el arte pop utilizó las mediatizaciones masivas como fuente de creación artística, pero exponiendo que se trataba de fenómenos publicitarios o culturales dentro de los medios masivos. 
Debe tenerse en cuenta, además, y en esto comenzamos a diferenciarnos de Van Dijck, que la vida mediatizada se ve diferente según el punto de vista de los intercambios discursivos que construye. Es que en las plataformas se producen o se practican, sin demasiados conflictos entre sí, diferentes tipos de intercambio discursivo: ver un mismo film en YouTube, en Netflix o en un canal de cable, si bien sitúa a los espectadores en muy diferentes sistemas de intercambio económico y hasta ecológico, no genera diferencia a la hora de compartir las diferentes interpretaciones que produjo la espectación ${ }^{\mathbf{5}}$.

Otros ejemplos: en plataformas con tanto networking como Facebook, desde cuyos muros se puede acceder a ese tipo de contenido de films o tutoriales colgados en la web, o libros enteros en PDF; o Twitter, que ha transmitido el Torneo de Tenis de Wimbledon y acaba de anunciar que va a emitir los encuentros de la NFL, esa clase de espectáculos televisivos en vivo que nadie discute como fenómeno de broadcasting. Por otro lado, un mensaje privado en Facebook dirigido a un colega profesional respecto de un congreso no es confundido con lo que debería ser la actividad básica en la plataforma: el contacto con amigos. Por esto pensamos que en el universo de las plataformas mediáticas, el estudio de los intercambios discursivos mediatizados debe recuperar su especificidad y su particularidad.

Para ir reconstruyendo esas particularidades, hemos definido recientemente la existencia de dos modos de participación en los intercambios mediatizados:

- La espectatorial, en la que los receptores tienen un lugar relativamente fijo frente al que les llega la emisión de su mediatización elegida y/o aceptada;

- Yla interactiva, sin duda, propia de las nuevas mediatizaciones, pero presente desde hace muchas décadas en los medios de sonido y en la gráfica de vía pública, oscilante en la prensa gráfica y con avances ahora en las mediatizaciones audiovisuales como el cine y las nuevas televisiones (Fernández, 2016).

Además de su interés descriptivo, la oposición espectatorial/interactiva permite comprender, por ejemplo, por qué suele molestar la presencia en Facebook y en Twitter de videos que se activan automáticamente: en un contexto interaccional, se introduce una propuesta espectatorial. No resulta agradable en nuestra cultura, salvo en ciertos contextos artísticos, que se pongan en evidencia diferencias en procesos materiales.

Como se ve, la oposición broadcasting vs. networking no se disuelve, pero se encuentran sistemas de intercambio diferentes dentro de lo que habitual-

5 No incluimos aquí la espectación en las salas cinematográficas porque tenemos en cuenta la descripción de Metz sobre los grandes "regímenes del significante cine" (Metz, 1979, p. 40) que incluían, no sólo el tipo de film, sino también su convivencia con otros objetos fílmicos y con la sala cinematográfica. En todo caso, un gran régimen del significante cine en las plataformas mediáticas o en los diferentes medios, debería incluir rasgos parecidos de espectación, todos distintos a los de la sala cinematográfica. 
mente se consideran las mediatizaciones masivas como opuestas en bloque a las mediatizaciones en red. Tenemos ahí una nueva oportunidad de formular parecidos y diferencias sin estar influidos ciegamente por el estilo de época que nos hace buscar novedades en cada detalle.

Ya enfocándonos en el mundo de las plataformas, vemos que está en expansión y en transformación constante: tengamos en cuenta que buena parte de las aplicaciones para smatphones son en sí mismas plataformas más o menos complejas, con diferentes relaciones con otras plataformas. Por ejemplo, la presencia de las transmisiones de la NFL en Twitter, ya mencionadas, generan un efecto de vértigo: en una plataforma central de micro-blogging -es decir, de producción y emisión de posteos más o menos individuales-, buscando rápida respuesta de los seguidores, aparecerá un clásico del broadcasting masivo: grandes eventos deportivos. El seguimiento y el comentario sobre eventos deportivos, muy comunes en Twitter, ahora podrá convivir con la recepción del evento en el mismo sistema de intercambio discursivo.

No importa aquí que lo descripto sobrelas relaciones entre Twitteryla NFL se materialice o que perdure exitosamente, sino que la incertidumbre sobre ese futuro convive con el riesgo de que la propia plataforma desaparezca por falta de rentabilidad e ingreso de capitales. Es decir, corremos el riesgo de que la lista de novedades nos impida ver los fenómenos que permanecen y que se van transformando a un ritmo propio, y con relativa independencia de otros fenómenos parecidos con los que conviven.

Siguiendo el modo que proponía Metz (1979) respecto de enfrentar el problema de la aparición constante de nuevas figuras retóricas, nos hemos propuesto para adaptarnos a esta nueva etapa de investigación, enfocarnos "en estatutos más que en la lista de novedades que cada día encontramos en nuestro recorrido mediático" (Fernández, 2010, p. 9).

Para introducirnos en la especificidad mediático-discursiva de las plataformas revisemos, aunque superficialmente, la sugerente manera en que José Van Dijck las clasifica. La investigadora holandesa, que tiene mucho cuidado en no parecer exhaustiva, organiza las plataformas de la siguiente manera:

Tabla 1: Tipos de plataformas.

\begin{tabular}{|l|l|}
\hline “Contacto interpersonal" & Facebook, Twitter, LinkedIn, Foursquare, etc. \\
\hline “Contenido generado por los usuarios" & YouTube, Flickr, Wikipedia, etc. \\
\hline “Comercialización" & Amazon, eBay, Groupon, MercadoLibre, etc. \\
\hline “Juegos" & Juegos online: Farmville, Angry Birds, etc. \\
\hline
\end{tabular}

Fuente: Van Dijck (2016, pp. 24-26).

La clasificación de Van Dijcksejustifica por el esfuerzo dela autora por relacionarlosociocultural con lo económicoy, desde ese punto de vista, resulta útil. 
Ahora bien, desde nuestro punto de vista, en ese artículo no se pueden discutir las categorías de plataformas de comercialización y las de juegos en el sentido de que, más allá de su diversidad, sólo se justificarían como plataformainterfaz para segmentos muy específicos de usuarios: para dispositivos utilizados para el trabajo o para niños que exclusivamente usen los intercambios digitales para jugar. Entre esos tipos de plataformas especializadas podrían incluirse también las educativas, que vienen siendo estudiadas hace tiempo (Traversa, 2009).

En cuanto a la categoría de las plataformas con contenidos generados por los usuarios, parece que a pesar de sus esfuerzos por parecerse a Facebook, Twitter sigue siendo básicamente un sitio de micro-blogging, en ese sentido compitiendo más con Instagram y Snapchat que son las que ganan terreno entre usuarios jóvenes. Todas estas plataformas tienen muchos más espectadores que productores, según las estadísticas disponibles. Tienden a constituirse, en ese sentido, en paralelo con el universo informativo de los medios masivos pero con presencia de individuos produciendo contenidos, aunque debe recordarse que son pocas las estrellas de estas plataformas que no tienen presencia también en el mundo del broadcasting.

En todas las plataformas de contenidos de usuarios, los posteos individuales tienden a competir con posteos corporativos y publicitarios. La presencia más importante del broadcasting (informativos, políticos, etc.) en Twitter se debe seguramente a su característica escritural. De todos modos, estas plataformas tienen una gran vida interaccional: cada posteo genera megusteos, comentarios y compartidos, y su ausencia llama más la atención que su presencia ${ }^{6}$.

Las plataformascentradas en las relaciones de redes como LinkedIn, Tindle o Foursquare y otras muchas, tienen dos barreras: para los que nos interesa aquí, son demasiado especializadas, cada vez más en competencia con aplicaciones que no dejan de aparecer y, por otra parte, tienden a ser absorbidas en sus funciones por las plataformas múltiples.

\section{PLATAFORMAS COMO INTERFACES}

En este capítulo trataremos un aspecto de las plataformas mediáticas que, si bien todavía no está plenamente instalado, desde el conocimiento que ya poseemos nos permite jugar con alternativas de futuro. Esto tal vez tenga importancia más centralmente para la industria; pero aquí pretendemos que contribuya a nuestra comprensión de los intercambios discursivos en época de plataformas.

6 Por supuesto que Twitter tienen mucha más complejidad en términos de sistemas de intercambio que las otras plataformas que mencionamos en este punto y que merece estudios especiales. Aquí, como se verá, nos interesa acentuar que si bien parece que, por un lado, Twitter trata de atacar a Facebook y, por el otro, defenderse de Instagram y Snapchat, es muy difícil pensar una escena de plat-faces (sintetizamos en este término el sintagma plataforma de interfaces) con la pantalla completa de Twitter, aunque por supuesto dentro de cualquiera una ventana de su time line. 
En un futuro cercano la convergencia tecnológica será plena, en el sentido en que tendremoslos mismos softwares y contenidos en nuestros smartphones, tablets, computadoras y televisores, con estosúltimos siendo talvez, solamente, la "pantalla grande" en el hogar. Este proceso se ve con claridad porque ya es muy fácil observar nuestro smartphone y nuestra computadora en la pantalla televisiva y también al revés: podemos ver buena parte de la emisión televisiva en otros dispositivos propios. Desde el smartphone yla computadora-que son los grandes dispositivos con los que producimos y distribuimos nuestros intercambios discursivos - todavía es algo sofisticado unir ambas pantallas, aunque ya vemos prácticamente la misma pantalla de las plataformas preparadas para funcionar en ambos.

Como se ve en las Figuras 1 y 2, hoy los usuarios podemos ordenar nuestras pantallas de inicio en smartphones y computadoras, con algunas de las siguientes características:

Figura 1. Plataformas cuasi-individuales.

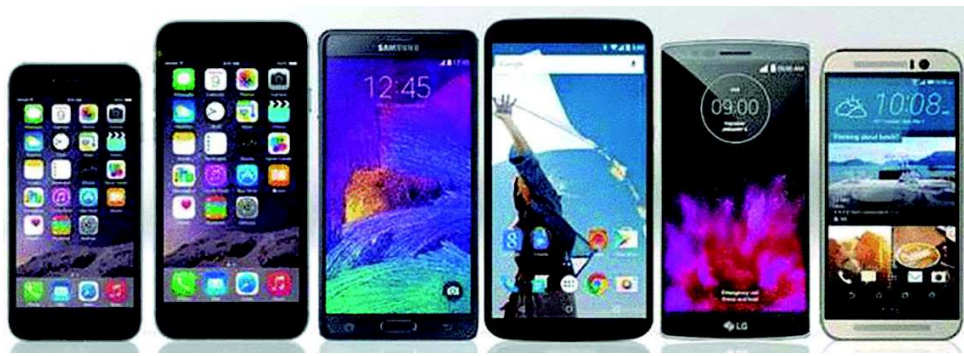

Fuente: elaboración propia.

Figura 2. Pantalla de computadora.

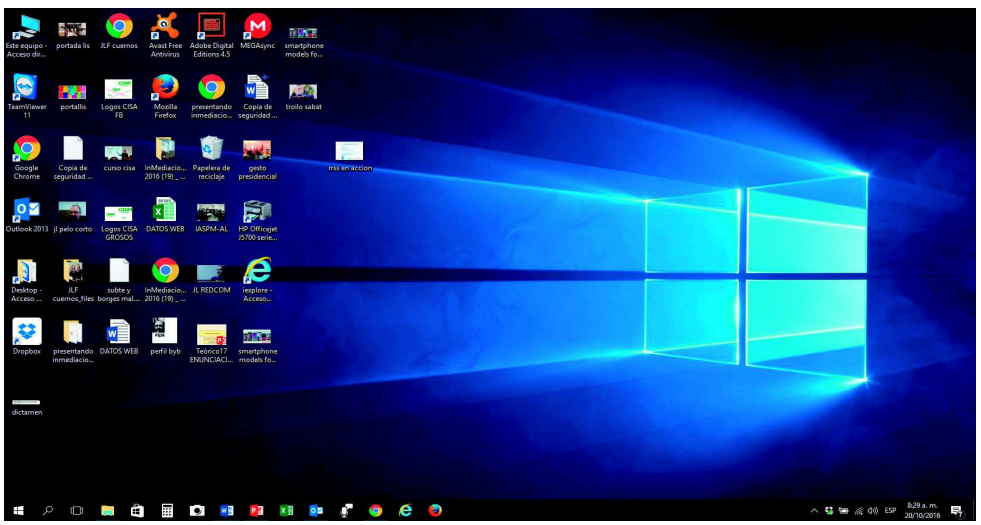

Fuente: elaboración propia. 
Estos diseños de interfaz están decididos por cada individuo pero en una tensión entre sus gustos, hábitos, necesidades y ofertas (y presiones), que los diversos diseñadores y desarrolladores de softwares y aplicaciones ofrecen. Estosinicios deberíamos entenderlos como nuestras plataformasinterfaz, porque permiten la copresencia de diferentes alternativas de intercambio mediático y de servicios. Un estudio detallado de los criterios aplicados nos diría mucho, tanto de los estilos de vida y usos de cada usuario, como también de estilos de intercambio discursivo; desde los que tienen que ver con el diseño, hasta los tipos de intercambio privilegiados.

Hay diversas posibilidades de aplicaciones o plataformas para cada necesidad; posiblemente muchas más delas que un individuo requiera en su vida: el ya típico efecto "hipermercado" en el que hay muchas más ofertas de productos y variedades de los que un comprador pueda siquiera imaginar, pero que genera ese apreciado efecto del deseo a generar y satisfacer. Y lo que se incrementará será la necesidad cada vez más compleja de articular las diversas posibilidades de una manera operativa.

¿Tal vez siempre haya soluciones individuales? Seguramente, pero hoy todavía nos resulta engorroso recorrer pantallas de nuestro Smartphone, o hacer zapping entre diversas ofertas y servicios de canales de TV, o combinar nuestros intercambios profesionales con los familiares, etc.

En el desarrollo de usos de algunas plataformas (por ejemplo, Figura 3) notamos un esfuerzo no demasiado explicitado aún por imponer sus pantallas como útiles para diversos usos. Es obvio que la visita y la permanencia dentro de cada pantalla y el linkeo son indicadores de éxito queluego serán argumento de venta de espacios publicitarios.

Figura 3. Muro (interfaz) de Facebook.

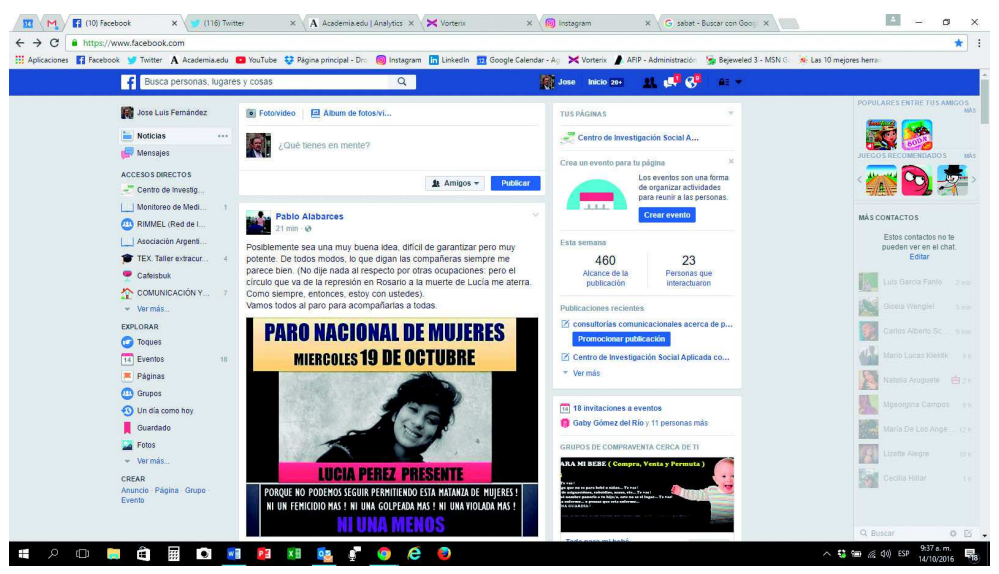

Fuente: elaboración propia. 
En ese sentido, cada interfaz de plataforma compite con las interfaces que diseña el usuario para organizar su trabajo y su navegación en las redes; así, Facebook nos permite vincular en un mismo espacio lo público y lo privado, lo laboral y lo lúdico, lo informativo y lo ficcional. Por supuesto que pueden agregarse (y se van a agregar) otros sistemas de intercambio, pero como seguramente ya hacen muchos usuarios, se puede iniciar un proceso de navegación a través de este tipo de pantallas. Aplicaremos aquí este enfoque proponiendo una tipología de plataformas a partir de ciertos estatutos básicos, pero enfocados en aspectos particulares: la posibilidad de constituirse en interfaces comunes, pantallas de inicio, de los diversos soportes de intercambio mediático que utilizamos: smartphones, las tablets, el televisores, las diversas computadoras portátiles o de escritorio que, como dijimos, compartirán nuestras mediatizaciones y contenidos.

Aquí enfrentamos un riesgo porque pareciera que nuestra línea de investigación nos lleva por caminos que no está marcando la industria, la que suele generar líneas de reflexión y de investigación académica que la soporten (o tal vez sea la vida académica la que trata de entender el futuro a través de las propuestas de la industria). De todas maneras, avanzamos en nuestro objetivo de estudiar plataformas que tienen, cada una, su importancia y particularidad, para comprender sus campos de interacción actuales y futuros. Y entendemos que esta línea de reflexión nos hace converger, aunque sea parcialmente, con preocupaciones centrales de la industria como expandir las visitas y su duración para generar espacios de mediatización más o menos estables en los que se pueda pautar publicidad en el sentido en el que se hacía en el broadcasting, o proponer directamente posibles intercambios comerciales a través del link con plataformas de venta.

Además, nuestra investigación se toca, de algún modo, con los temas de supervivencia de las plataformas y como se verá, si bien se hablará de marcas privilegiadas en cada caso, la idea es pensar a cada una de esta categorías como familias de plataformas que podrán realizar en el futuro alianzas de filiación.

\subsection{PLAT-FACES INFORMATIVAS}

Las plat-faces informativas son las plataformas que tienden a complejizarse y son las originadas en medios masivos o sus competidores exclusivamente on-line (diarios, canales de TV, radios). Van Dijck no las tuvo en cuenta, posiblemente porque se las puede considerar del lado del broadcasting. En ellas, en las plat-faces informativas, la tendencia a proponer entradas a más y muy diversos sistemas de intercambio, pero podrían reemplazar, desde el inicio de los diversos dispositivos, a aquella actividad que muchos individuos modernos practicaban al comenzar la jornada: escuchar radio mientras leían al diario. Puede verse en el caso de The Guardian (Figura 4) cómo la presencia central de 
publicidad del gobierno dela Ciudad de Buenos Aires, en Argentina, desmiente el enfoque central de la plataforma de un diario británico con pretensiones de globalidad. Se trata de un caso, frecuente, en que la geolocalización pone en cuestión a la globalización y a la red.

Figura 4. The Guardian.
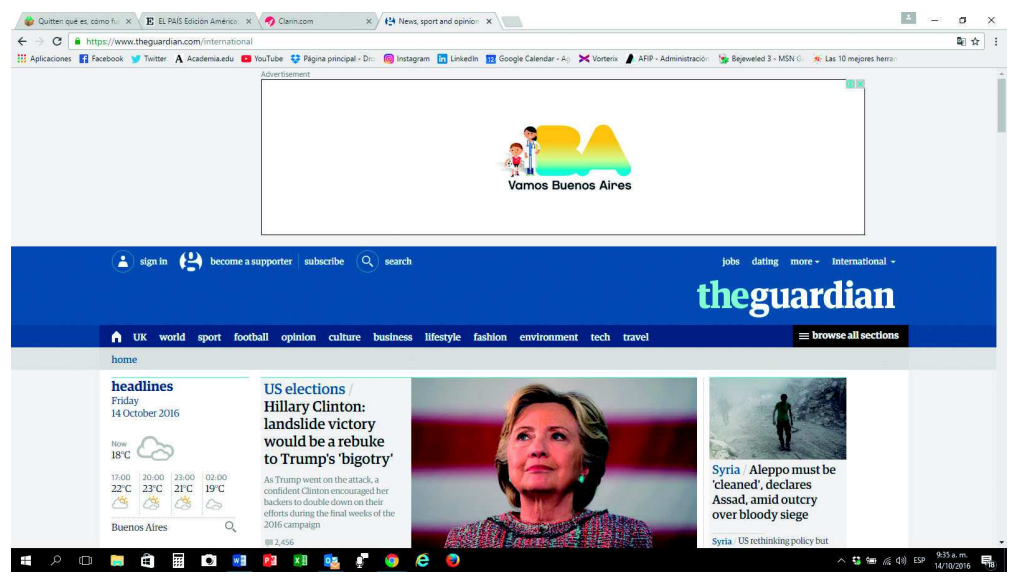

Fuente: elaboración propia.

Es curioso pero los grupos mediáticos no tienden a generar plataformas de grupo (Grupo Prisa, Grupo Clarín, el propio The Guardian, etc.), tal vez porque no se ha advertido esta posibilidad de uso, o porque están pensando, todavía, modelos de negocio previos. Tal vez la plataforma de la $\mathrm{BBC}$ sea la que más se parece a una interfaz múltiple, pero enfocada en quienes pretenden comenzar el día desde la información general.

\subsection{PLAT-FACES LOGÍSTICAS}

Las plataformas basadas en browsers (Google Chrome, Mozilla, Bing, etc.), tienden cada vez más a resolver la organización de la vida cotidiana y profesional: agendas de actividades, mails, notas. Ideales para el usuario profesional quien, como primera actividad del día, revisa actividades, correo, propuestas, etc.

Vemos que en este caso, la interfaz articula amigablemente la pantalla central con las herramientas organizadoras y soporte de actividades, muchas que son propias de la vida mediática, pero otras relacionadas con áreas de la personalylaboral. Además ofrecelink directo a sitios frecuentados por el usuario. Por último, el doodle (Figura 5), convertido prácticamente en un género gráfico-celebratorio, sitúa al usuario en la región en que está localizado, pero desde una apelación a su contexto cultural. 
Figura 5. Google Chrome.

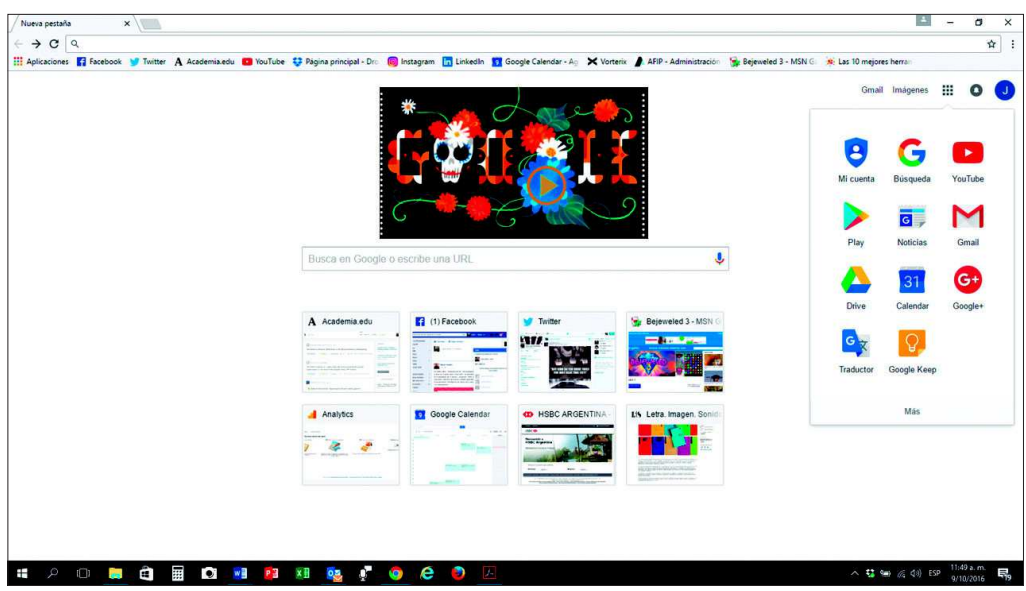

Fuente: elaboración propia.

\subsection{PLAT-FACES BROADCASTERS}

Denominamos plat-faces broadcasters a los sitios en los que se ofrecen diversas ofertas para recepción espectatorial. En ese sentido, las que presentamos al hablar de las plat-faces informativas se especializan precisamente en la información; mientras que aquí, el caso de Vorterix.com que venimos estudiando nos muestra que no sólo se puede articular radio con televisión, sino también audio con video, y lo informativo duro con lo documental y hasta lo ficcional.

Por supuesto que es difícil considerar como importante una plataforma originada en un país como la Argentina, cuyo idioma es exclusivamente el español, pero a pesar de haber explorado y consultado en otros países considerados innovadores, no hemos encontrado una plataforma equivalente. Es ese sentido, sigue siendo un ejemplo válido de plataforma con un desarrollo específico. Vorterix.com combina intercambios mediáticos en vivo y en broadcasting, combinados con espectáculos musicales en vivo y la presencia de una grilla de celdas discursivas, mayormente audiovisuales (Figura 6). Si bien cada celda discursiva está más habitualmente vinculada a la música que a otros tipos discursivos, puede sostenerse como modo de uso y organización del ingreso al sistema de intercambio mediático para quienes prefieran lo informativocultural, por sobre lo profesional o lo interindividual. 
Figura 6. Vorterix.com.

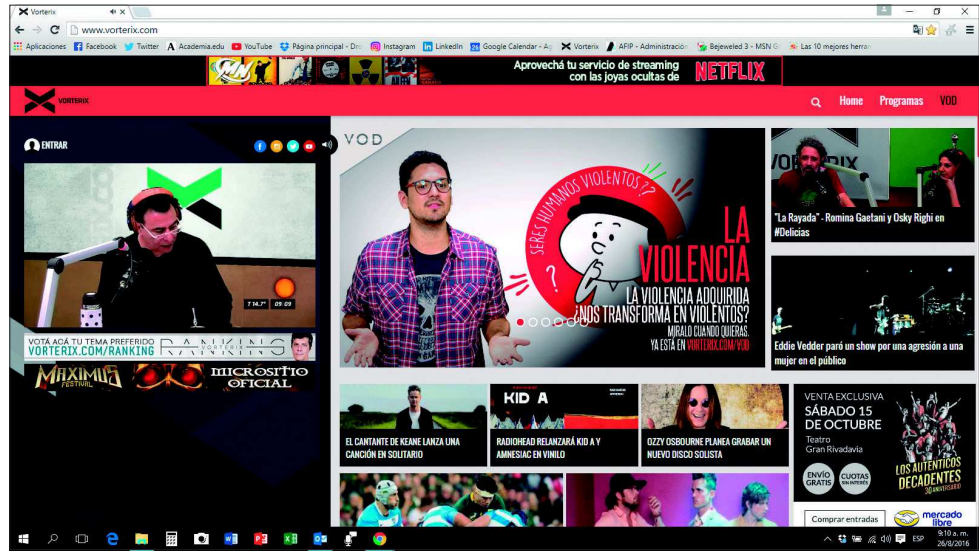

Fuente: elaboración propia.

En este campo, la fuerza de las clasificaciones mediáticas y la búsqueda de modelos de negocios rentables y claramente enfocados seguramente han impedido combinar las sinergias posibles entre los universos informativos y ficcionales. Llama la atención, en ese sentido, cómo plataformas exitosas como Netflix sólo se han concentrado en la ficción, y Spotify exclusivamente en la música, sin volcarse hacia, por ejemplo, los videos musicales, la radio en vivo o el podcasting. Este es otro campo de interés para profundizar a partir de lo que se ha denominado como narrativas transmedia. Si bien ha tenido, en un principio, más desarrollo en la reflexión sobre la combinatoria de relaciones entre medios y plataformas, en la entrevista a Gonzalo Frasca publicada en el libro Narrativas transmedia. Cuando todos los medios cuentan (Scolari, 2013) se habla de las relaciones entre gamificación y periodismo. Las narrativas transmedia comienzan, entonces, a ser también transgénero o, con más precisión, transtipos discursivos. De todos modos, Vorterix.com merece ser considerada como multimodality o, simplemente, como multimedia: no mezcla, sino que articula medios y géneros, contribuyendo con esa estrategia a la vida cultural transmedia.

\subsection{PLAT-FACES DE INTERACCIONES MÚLTIPLES}

Seguramente ya hay muchos usuarios que lo primero que hacen al conectarse es comenzar su vida mediática en red y por Facebook (por supuesto, otros lo harán con otras redes), pero creemos que alrededor de Facebook hay un gran malentendido, en buena parte atribuible a los propios administradores de la 
plataforma: el seguir considerándola como una "red de amigos" o, algo más extensamente, como una red de contactos ${ }^{7}$. Observemos entonces su pantalla:

Figura 7. Facebook.

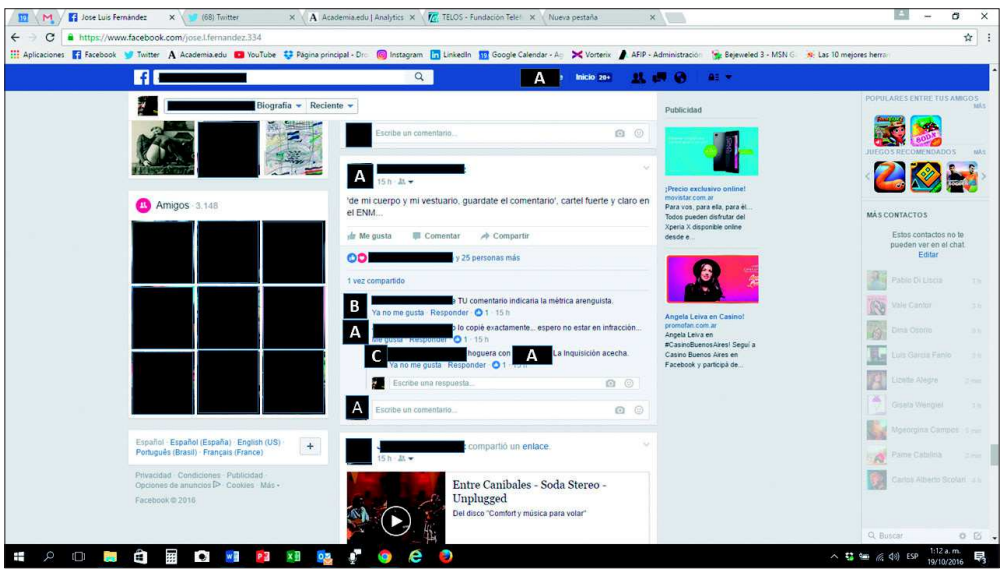

Fuente: elaboración propia.

Si realizamos un recorrido sobre lo que se observa, vemos que se combinan sistemas de intercambio privados con semipúblicos, organizaciones de agenda con informaciones tipo time-line de Twitter, sistemas de juegos y de relaciones con grupos, contacto con pautas publicitarias o con eventos organizados más allá del interés individual. Es evidente que la presencia de medios broadcasters, que completaría el conjunto de sistemas de intercambio más habituales, no se basa todavía en acuerdos directos con los medios que permitan acceder directamente a sus contenidos. Pero a través de sus páginas, en el propio Facebook pueden organizarse sistemas de información, y combinando con YouTube, hasta de recepción de filmes de duración prolongada. Por lo tanto, en esta familia (Facebook es el integrante más conocido en la actualidad) de plataformas equivalentes, parecen ser los plat-faces más complejos y con más posibilidades de desarrollo.

No creemos que altere este nivel de análisis, de todas maneras provisorio, dos aspectos presentes: que estas plataformas permiten cierto grado de edición por cada usuarios y que sus interfaces en smartphones son algo más sencillas que en tablets y computadoras. El fenómeno, en general, creemos que es el mismo.

7 Los rasgos diferenciales de Facebook como ámbito que excedía el simple contacto entre amigos lo vio muy temprano Alejandro Piscitelli, quien con su equipo de cátedra en la Facultad de Ciencias Sociales de la UBA hicieron una experiencia de cátedra en Facebook ya en 2009 (Adaime 2010). 


\section{LO MICRO EN LO MÉDIUM: EL ANÁLISIS SOCIOSEMIÓTICO EN INTERCAMBIOS ESPECÍFICOS}

Vamos a mostrar aquí la necesidad de hacer estudios micro, en el mismo momento en que estamos discutiendo la problemática de las interfaces, lo cual es en este sentido, y tal como lo advertimos en nuestra Introducción, un tema médium. Si en el enfoque de las plataformas entre sí, al menos en un primer análisis, solemos privilegiar a la ecología de los medios, al tratar de comprender el funcionamiento detallado de cada una de ellas debemos analizar fenómenos micro, muy diferentes entre sí: prestar atención a estos intercambios micro nos permite ver la extensión atemorizante que deberán tener en el futuro los estudios sobrelas mediatizaciones, sino se quiere que tenganla pobrezafinalde gran parte del conocimiento que se generó sobre las mediatizaciones masivas.

Los tres casos que vamos a revisar superficialmente son solamente algunos, entre muchos posibles e incluso de mayor importancia. Los elegimos porque no tenemos duda de que son frecuentes y porque, además, representan bien lo que queremos justificar en este trabajo. Estos casos son:

- La recepción espectatorial de films, más allá de la plataforma a través de la que se acceda a ellos;

- La sucesión de comments: participación en largas secuencias de posteos y comments relacionados, especialmente en Facebook, pero posibles en otros tipos de plataformas, como Twitter, WhatsApp o Telegram;

- Un fenómeno muy particular pero reconocido por todoslos usuarioshabituales de Facebook que son los megusteos diferentes, presencia en una actividad aparentemente muy sencilla y habitual de gestos que se consideran con una significación especial.

Aunque tienen estatutos diferenciados, los tres podrían realizarse a través de Facebook, y ello en parte justifica nuestro interés, luego del lugar como platfaz que sugerimos para la plataforma.

El primero, la recepción espectatorial, en tanto espectación en broadcasting, es poco considerada porque es equivalente a fenómenos de la mediatización masiva. El segundo, la sucesión de comments, es prácticamente el que se considera central en las vidas de las plataformas entendidas como redes, en networking. Como se ha visto, para nosotros es uno más, aunque importante, entre muchos otros posibles. El tercero y último, el de los megusteos diferentes, es una especie de efecto lateral, micro dentro de lo micro, y que, sin embargo, forma parte de lo más profundo de la comprensión interaccional de Facebook como red de contactos.

8 La observación de la importancia de este tipo de recepción y la noción de celdas nos viene de nuestros estudios sobre Vorterix (Fernández, 2014) y la importancia que le otorgábamos allí a la construcción en grilla de celdas. 
Cuando sellega a este nivel de estudio en detalle, se presupone que yahemos superado etapas previas de investigación. Sin pretender agotar las posibilidades de análisis, en un trabajo reciente (Fernández 2016) proponíamos que para comprender claramente estos intercambios tan particulares ${ }^{9}$ debíamos tener resueltos, aunque más no sea parcialmente, los dos momentos previos. El primero, una reconstrucción del set de ofertas mediáticas disponibles para quien participa de estos intercambios (competencia entre plataformas); y el segundo, un análisis comparativo del uso habitual entre esas plataformas que hace cada individuo estudiado (hábitos de uso de los diferentes modos de intercambio quele permite la plataforma que se estudia). Si no, como ha ocurrido en muchos estudios sobre los medios masivos, se corre el riesgo de tomar como excepcional algo que un usuario hace habitualmente y que, por lo tanto, debe ser explicado en un nivel superior de análisis.

\subsection{EL ANÁLISIS DE CELDAS DISCURSIVAS}

Dijimos que íbamos a analizar la recepción de filmes, pero sólo para simplificar la presentación. Prácticamente en cualquier plataforma mediática tenemos la posibilidad de acceder a algún texto, de cualquier mediatización de origen, y al cual podemos recorrer sin alterar su secuencia original: un film de ficción o documental, un aviso publicitario, un artículo de difusión o de origen científico, un tema musical con su respectivo video de soporte, un tutorial sobre una receta de cocina o una tecnología, un capítulo Wikipedia, etc. La hipótesis es que en esa circunstancia no hacemos nada demasiado diferente a ver un film o un video a través de un aparato televisivo, o leer una revista o un libro. Se trata entonces, de celdas de intercambio espectatorial insertas en plataformas interaccionales: típicos y frecuentes casos de intercambios en plataformas más allá de las redes.

No pretendemos profundizar demasiado pero sobre esos intercambios, al menos en principio, deberían aplicarse todas las metodologías de los que denominamos sociosemiótica de las mediatizaciones. Es decir, primero deberíamos comprender la historia de esos dispositivos técnicos ${ }^{\mathbf{1 0}}$, sus relaciones con los géneros y estilos que intervienen en esa discursividad y estableciendoluego los usos sociales a los que se aplican en general (semiohistoria). Luego deberíamos poder describir cómo están construidos esos textos en ese cruce genéricoestilístico, pero teniendo en cuenta sus vidas en diferentes mediatizaciones y

9 Intercambios a los que denominamos intercambios discursivos extensos (por supuesto que no por su extensión material, sino por la necesidad de aplicar modelos complejos de análisis mediático y discursivo).

10 No tiene sentido aquí profundizar el tema, pero cuando decimos dispositivo técnico nos referimos al resultado material que las tecnologías aportan a la construcción discursiva: no es el papel, sino el efecto de permanencia y de posibilidad de recorrido oblicuo; no es el parlante sino el efecto de transparencia en el transporte del sonido, ni la pantalla, sino su efecto de ventana ópticamente indicial hacia un referente. (Fernández, 2012). En cambio cuando decimos dispositivo smartphone, sólo hablamos del aparato como opuesto a la computadora. En ambos casos, no aplicamos nociones de dispositivo como conjunto de reglas o costumbres sociales. 
sus transposiciones. Y, por último, realizar las operaciones de análisis de textos habituales en la semiótica de los medios (figuraciones temáticas, retóricas y enunciativas, usos estilísticos de los dispositivos técnicos, etc.).

La única diferencia, importante pero no desestructurante, es que esos análisis pueden relacionarse muy rápidamente en las plataformas multimedia con resultados de reconocimientos y hasta, frecuentemente, con relaciones con comments propios o de otros receptores. Se dirá, desde un sentido común de época, que esto último es lo realmente importante; nuestra respuesta será que eslo diferencial pero que no hay relación en extensión cuantitativa entre vistasy comments. Siempre aquellas superan a las señales de reconocimiento y siempre los textos son el nodo de esos intercambios discursivos.

\subsection{EL ANÁLISIS DE LAS SECUENCIAS DE COMMENTS}

Una primera inspección sobre estos tipos de intercambio nos anuncia que estamos en el mundo de la conversación o, para ser más precisos y por la extensión actual del fenómeno, en el mundo del chat. Es verdad que los intercambios en los muros no son, o no son con mucha frecuencia, chats interindividuales, pero WhatsApp, con su facilidad para armar grupos, ha extendido esta forma de intercambio escritural. Y lo primero que debemos preguntarnos es: ¿por qué los estudios dedicados a la conversación no convergieron sobre los fenómenos del chat y sus derivados? No es aquí el lugar para profundizar sobre las fuerzas y debilidades de los estudios sobre la conversación, aunque habría que explicar por qué esos estudios no siguieron su desarrollo, lo cual se debe, creemos, más a una falta de impulso de investigación que a las diferencias propias entre conversación hablada y conversación escrita.

La conversación es una tipo discursivo, en principio no mediático, que sin embargo es nuclear en/de la práctica telefónica, mediática aunque interindividual, desde fines del siglo XIX. Los estudios sobre la conversación se desarrollaron a partir de la década del 60 del siglo XX y sus orígenes no provienen de la lingüística, sino de diferentes ciencias sociales y de la filosofía (Tusón Valls, 2002). Nunca pudieron aplicarse a las conversaciones telefónicas por la necesidad de autorización de los intervinientes y el consiguiente riesgo de sesgo. Ello ha privado a los estudios sobre las mediatizaciones de antecedentes de intercambios en red de largo recorrido.

Otra dificultad para avanzar en los estudios conversacionales era que obligaban a complejos trabajos de transcripción para pasar de lo oral a lo escritural académico, también obligaban a complejas operaciones descriptivas de reconstrucción delos contextos alos que obliga todo fenómeno pragmático, como estas interacciones verbales tan extensas.

Frente a la complejidad de la conversación verbal, los intercambios escriturales en los muros de Facebook (o en la TimeLine de Twitter) tienen varias ven- 
tajas ${ }^{11}$ la principal es que las capturas de pantalla permiten recoger el contexto directo donde se desarrolla el intercambio a estudiar y el propio intercambio aislado o recortado (Figuras 6 y 7 ).

Enla Figura 8 se puede reconocer que el intercambio ocupa un lugar central, pero en competencia con el conjunto de la interfaz de Facebook. Esto se debe sólo en parte a que es un intercambio breve: la página de la plataforma ofrece múltiples posibilidades de distracción o de acción lateral. Es decir que cuando un usuario registra y participa en estos intercambios debe otorgarle al propio intercambio algún rango de importancia.

Figura 8. Muro de A en Facebook

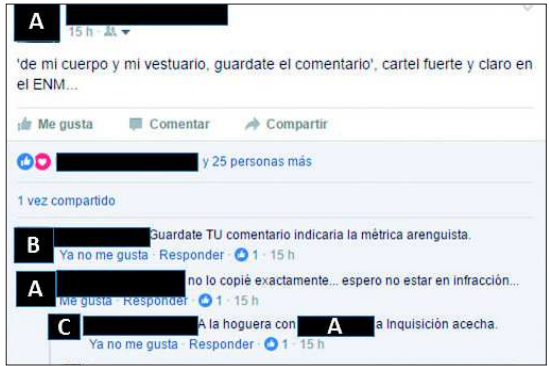

Fuente: elaboración propia.

Analicemos ahora el desarrollo de las relaciones entre el posteo original y sus pocas repercusiones (Figura 9).

Figura 9. Intercambio destacado para ser analizado

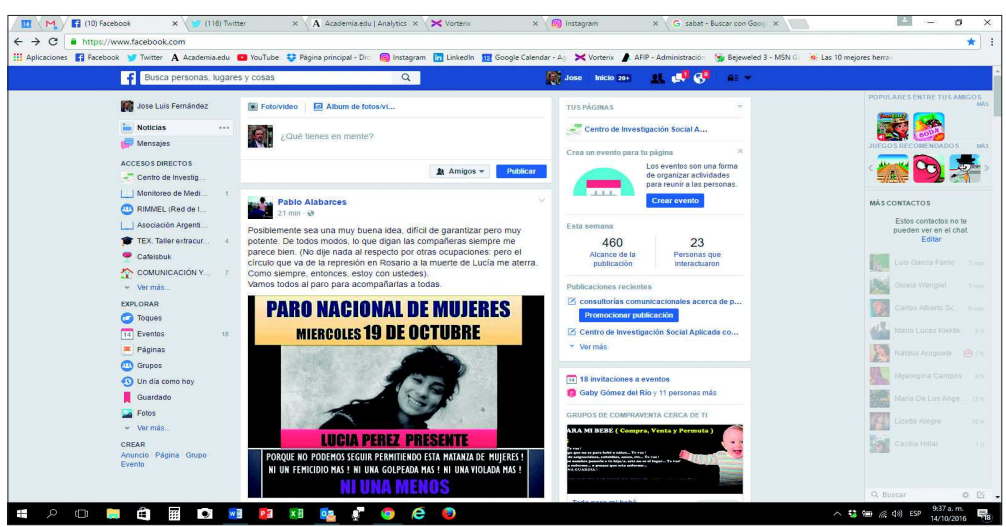

Fuente: elaboración propia.

11 Frente a la protección legal de las conversaciones telefónicas como dentro de los chats interindividuales, el juego entre posteos y comments en los muros de Facebook, si bien pueden ser bloqueados, en general son, al menos, cuasi-públicos. 
El objetivo será entrever la complejidad puesta en juego y no profundizar su análisis:

Posteo de A: 'de mi cuerpo y mi vestuario, guardate el comentario', cartel fuerte y claro en el ENM...

En primer lugar, se registra la referencia a un cartel y al Encuentro Nacional de las Mujeres (ENM) realizado en octubre de 2016 en la ciudad Rosario, Argentina. Es decir que este posteo, publicado en una plataforma global, aunque sólo para amigos de Facebook (una posibilidad más restringida que público), se inscribe en un momento muy activo en torno de los conflictos de género: en una semana se realizó el ENM y, algunos días después, una movilización denominada Miércoles Negro delucha en contra dela violencia contrala mujer, todo enmarcado en femicidios frecuentes y brutales ${ }^{12}$. El posteo tuvo 25 megusteos e interacciones con predominancia femenina, es decir que se sintonizó al menos en algo con actores activos del conflicto ${ }^{13}$. Un factor a tener en cuenta, y que obliga a una interacción etno-semiótica, es que entre los megusteadores, A conoce a alguno de la vida no virtual, a otros de la virtual y otros son apenas contactos. Como veremos luego en los megusteos diferentes, éste es un dato clave. De todos modos, a pesar de la brevedad, el posteo aporta un referente complejo y poco precisado (se requiere un tasa de presuposición común bastante alta para entenderlo), yademás lo cruza con un componente de evaluación siempre riesgoso en momentos de sensibilidad temática y movilizadora.

Posteo de B: Guardate TU comentario indicaría la métrica arenguista.

Este primer comment hace referencia en tono juguetón a cómo debería adaptarse el slogan reproducido a la oralidad de la consigna cantada, si fuera cantada. B es alguien que comprendió perfectamente de qué se trataba el posteo y, en ese tono criticón, pero no serio, frecuente en las redes, propone una corrección. Contexto, posteo y comment puede decirse que comparten el mismo espacio desde el punto pragmático. El arenguismo agrega un tono de cercanía y se presupone un rasgo de sofisticación respecto de los lenguajes en correlación con el rasgo evaluatorio del posteo.

12 Ese conflicto local se reprodujo con movilizaciones en el mismo miércoles en muchas ciudades y países de Latinoamérica.

13 Una objeción posible que se le puede hacer a este ejemplo u otros similares es que "todo está filtrado" por el algoritmo de Facebook que limita las relaciones. No es aquí el lugar para discutir un tema tan complejo; pero sí para advertir que sin enfoques micro sobre estos intercambios, los datos de algoritmos y big-data podrán ser aplicados, pero con enormes dosis de imaginarización que están fuera de control para todos los interesados. Por ejemplo, son muy interesantes los análisis de Rudder (2016) sobre la base de datos y la actividad de sus usuarios; pero es una plataforma muy especializada, de contactos interpersonales para salir, en la que se deben dar datos de perfil más o menos ciertos, no sólo sobre el propio, sino sobre los del contacto buscado; información compleja, pero, por decir así, mononivel. De todos modos, ese principio organizador será fuertísimo si se combina, como ya se estará haciendo, con otras bases de datos. Se verán los resultados. 
Nuevo posteo de A: No lo copié exactamente... espero no estar en infracción...

Esta respuesta, en cierto sentido, cambia la escena: en la unicidad escasamente conflictiva, detectada en un juego casi técnico con el lenguaje, se introduce una disculpa sobre la precisión posible de la cita y aparece también el riesgo de la sanción frente a un error -aquí para la comprensión se necesita incorporar una presuposición más sofisticada sobre el contexto, ya que en realidad había tendencias en esas movilizaciones relativamente anti-varoniles que generaron discusiones, en el extremo, sobre si los varones debían participar o no-.

Aquí aparece un rasgo irónico con el habitual riesgo que la ironía tiene en toda conversación y que en las redes suele aumentado por la falta de interacción gestual o proxémica.

Posteo de C: A la hoguera con A! La Inquisición acecha.

La intervención de $\mathrm{C}$ profundiza el toque irónico de $\mathrm{A}$. Esto resulta algo atenuado porque la respuesta no es en el nivel de la secuencia general sino, como permite desde hace un tiempo Facebook, responde a A sin incluir necesariamente a B. De todos modos, los que acceden a la conversación pueden acceder a este comment, pero también acceden a su señalización gráfica. Lo cierto que aquí, el juego de interacciones, para ser realmente comprendido, debe incluir la convocatoria a centenarias formas de represión, con un tono irónico que, dada la conflictividad del momento, pueden desembocar en una fuerte discusión con otros que pueden estar siguiendo el intercambio.

A pesar de la superficialidad de nuestro análisis se ve, en primer lugar, la complejidad de este fenómeno breve y acotado. También creemos que con este ejemplo se puede registrar que, para comprender el funcionamiento de este muro y de este usuario, hace falta analizar al menos una muestra representativa de la sucesión de posteos para establecer un principio estilístico ordenador. Además, el análisis obligará a revisar constantemente al menos cuatro capas superpuestas de contextos: la de la secuencia que, cuanto más extensa es, más obliga a controlar la linealidad o la arborescencia de la misma; las relaciones con el conjunto del muro; las referencias al contexto social directo y, por último, la del universo de gramáticas de reconocimiento requerido para que el intercambio, primero exista, y luego que se pueda comprender su campo conflictivo. Estos análisis son una tarea sólo posible en un largo lapso de tiempo y realizada por un equipo con muchos investigadores ${ }^{14}$.

14 La investigación citada de Miller (2016), que es muy rica en conclusiones sobre este tipo interacciones entre individuos o grupos de individuos, fue realizada mediante observaciones participantes en ocho ciudades por diferentes investigadores, residiendo quince meses en cada ciudad estudiada. 


\subsection{EL MEGUSTEO DIFERENTE. MÁS ALLÁ DE LO ETNOGRÁFICO YEL BIG DATA}

Estefenómeno es muy particulary sin duda su inclusión aquí puede resultar conflictiva. En términos generales el megusteo es considerado, y con razón, como una acción y no como un fenómeno discursivo. El megusteo tiene un tono acumulativo y cuantitativo equivalente al levantar la mano para aprobar o desaprobar en una votación grupal cara a cara. Sin embargo, absolutamente todo usuario activo consultado reconoce que son frecuentes megusteos diferentes. Algunos megusteos se recortan frente al conjunto e introducen una presencia otra que obliga a la interpretación o que, muchas veces, carga con una interpretación previsible: seducción, ganas de llamar la atención, interés, voluntad de mostrar una presencia constante $\mathrm{u}$, otras, con interpretaciones muy selectivas.

La pregunta aquí es: ¿cómo estudiar el fenómeno para comprender cuáles son los criterios del usuario para registrar la diferencia y cómo establecer pautas de interpretación que no respondan exclusivamente al mundo imaginario o al deseo del usuario estudiado? Como se ve, a partir de lo micro, en los tres casos que proponemos, encontramos preguntas que, según sea la respuesta, cambiarán completamente la visión macro sobre las mediatizaciones.

Desde nuestro punto de vista, los megusteos han sido tratados hasta ahora como casos de interacciones particulares. Es decir, como relaciones de presencia / no presencia ${ }^{15}$. Pero el reconocimiento por los usuarios de una variedad de referentes para un signo siempre igual (y sencillo) obliga a considerarlas como interacciones extendidas, o sea, interacciones de tipo simbólico y con una complejidad equivalente a cualquier intercambio discursivo.

¿Cómo estudiar a los megusteos diferentes en tanto que interacciones extendidas? Suponemos que los lectores especializados ya están pensando en el poder de los análisis cuantitativos de big-data desde los cuales se promete comprender todos los fenómenos de gran extensión cuantitativa. Pero aquí encontramos uno de los grandes puntos de interés de los megusteos diferentes, tanto como fenómeno complejo como respecto a los límites y fuerzas de las metodologías. Desde el punto de vista metodológico, el click que genera la aparición de un megusteo común no se diferencia en nada de los movimientos del megusteo diferente. La diferencia, esa que registra el usuario, sólo puede ser comprendida por el sistema de relaciones tanto textuales, como intertextuales, en las que se inscribe. ¿Se podría construir patrones con Big Data? Sin duda, pero resulta difícil bajarlos a la realidad mediática sin consultar con los que construyen los usuarios. Si no, podría deberse esos patrones a fenómenos de otro nivel.

Elestudio de los megusteos diferentes, comolo proponemos aquí, sólo puede realizarse mediante la reconstrucción de grandes series de presuposiciones

15 La propiaplataforma ha reconocido la presencia del megusteo como algo limitado, por eso ha agregado al emoji del dedo levantado, otros propios del encanto, la tristeza, la risa y la sorpresa. La oposición entre interacción particular e interacción extendida está detallada en Fernández, 2016. 
etnográfico-semióticas. Como tantos fenómenos de la vida social, ese funcionamiento que cada usuario hace, con mayor o menos precisión, con mayor o menos consistencia, pero de una manera casi automática, requerirá de largas jornadas de estudios presenciales sobre el funcionamiento en Facebook de cada individuo estudiado. En términos de Lévi-Strauss (1977), largos procesos de construcción de modelos mecánicos para, en algún momento, y mediante trabajos indudablemente interdisciplinarios, acceder a modelos estadísticos.

\section{CONCLUSIONES: OTRA VEZ DE LOS MICROA LO MACRO}

En lo que hemos denominado como tercer momento de los estudios sobre las nuevas mediatizaciones, comienzan a destacarse fenómenos originales o movimientos que antes no existían o que, como otros de las mediatizaciones previas, pasaban desapercibidos. Entre los fenómenos a los que se les prestó atención en este artículo se destacan las plataformas mediáticas complejas, en las que se producen intercambios muy diferentes entre sí. Muchos de esos intercambios no son propiamente intercambios en red (networking), hasta el punto que aparecen como residuos resistentes y saludables del broadcasting.

Se ha sostenido aquí que el funcionamiento en plataformas excede a la presencia de plataformas prediseñadas. Las organizaciones de la interfaz en smartphones, computadoras, tablets y televisores son un ejemplo de organizaciones de interfaz en plataforma, en tensión entre la oferta mediática y la demanda de uso. Otro caso interesante de plataformas de uso mencionado es la escucha a través de auriculares, como espacio de switcheo entre comunicaciones masivas como las de la radio, broadcasting en red como el podcasting y los diversos tipos de playlists, más o menos diseñados por el otro y los mensajes de voz. Aquí se genera la posibilidad de interactuar desde el smartphone en diversos sistemas de intercambio, que van de lo masivo a lo interindividual.

Esta nueva atención sobre las plataformas mediáticas permite, a su vez, reflexionar y comenzar a investigar ciertas plataformas particulares, especialmente complejas, cuyo diseño múltipleles permitiría competir, con fuerzas y debilidades, con esa tendencia que aparece en los usuarios a personalizar la interfaz de base de todas las pantallas. Con ese objetivo se describieron las características de las familias de lo que hemos denominado plataformas de interfaz (plat-faces): las de baselogística, las depreponderancia informativa, las de preminencia en diversidad de broadcasting y las más poderosas de intercambios en múltiples niveles y tipos.

Si la investigación sobre plataformas construye objetos de estudios extensos en un nivel de los que hemos denominado médium, la investigación de esas plataformasponeenevidencialanecesidaddeindagartambiénenfenómenosmicro de intercambios discursivos que las constituyen. A esos intercambios los hemos denominado como extensos por la complejidad fenoménica y los requerimientos metodológicos a los que obligan, a pesar de su superficial sencillez o lateralidad. 
Se ha analizado con el ejemplo de la recepción de celdas discursivas espectatoriales que requieren la aplicación de técnicas de investigación sociosemióticas y de análisis del discurso. También se observaron las secuencias de posteos y comments que obligarán a volver a los estudios de pragmática sobre sistemas conversacionales. Por último, se ha ejemplificado la compleja presencia de un fenómeno como los megusteos diferentes, para lo que hemos propuesto recurrir a una crítica semiótica del gesto de megustear, y a la obligación, para su comprensión, de estudios detallados delos sistemas de presuposición delos diversos casos estudiados.

Este modo de estudio de los fenómenos micro y las metodologías propuestas, pero no cerradas, muestra la explosión de recursos humanos, técnicos y temporales que se requerirán para entenderlos desde su base material y evitar la ignorancia social que surge de la generalización y el aplicacionismo teórico.

Entrevemos un futuro en el que gran cantidad de equipos - de una conformación más parecida al de la integración de talleres, observatorios, monitoreos o workshops- que trabajen coordinadamente y al mismo tiempo desde diversas instituciones. A partir de ello tal vez y por fin se haga realidad la unión permanente entre enseñanza einvestigación. Otravez, serála fuerza dela transformación social micro y su reconocimiento la que cambie a las instituciones académicas. Y, en esa sinergia, acaso las instituciones de enseñanza y aprendizaje estén por fin en relación cercana con la transformación social.

\section{REFERENCIAS}

Adaime, I. (2010). El Proyecto Facebook y la creación de entornos colaborativos educativos. En Piscitelli, A., Adaime, I. y Binder, I. (compiladores). El proyecto facebook y la posuniversidad. Sistemas operativos sociales y entornos abiertos de aprendizaje (pp. 21-34). Madrid: Ariel.

Fernández, J. L. (2010). Listas y estatutos en las novedades mediáticas. En L.I.S. Letra, imagen, sonido. Ciudad mediatizada, 5, pp. 6-8.

Fernández, J. L. (2012). La captura de la audiencia radiofónica. Buenos Aires: Líber Editores.

Fernández, J. L. (2014). Mediatizaciones del sonido en las redes. El límite Vorterix. En Rovetto, F. \& Reviglio, M. C. (compiladoras), Estado actual de las investigaciones sobremediatizaciones (pp. 190-206). Rosario:UNREditora. Recuperado dehttp:// www.cim.unr.edu.ar/archivos/cuadernodelcim2.pdf

Fernández, J. L. (2015). ¿Una sola comunicación masiva? En L.I.S. Letra, imagen, sonido. Ciudad mediatizada, 13, pp. 10-14.

Fernández,J.L. (2016). Interacción: un campo de desempeño múltiple en broadcastingy en networking. En Cingolani, G. \& Sznaider, B. (editores), Nuevasmediatizaciones, nuevos públicos (pp. 10-26). Rosario: UNR Editora. 
Gitelman, L. (2006). Always Already New: Media, History, And The Data Of Culture. London: MIT press.

Jenkins, H. (2008). Convergence Culture. La cultura de la convergencia de los medios de comunicación. Barcelona: Paidós.

Joseph, I. (1999). Erving Goffman y la sociología. Barcelona: Gedisa.

Lévi-Strauss, C.(1977).La noción de estructura en etnología. En Antropologíaestructural (pp. 299-338). Buenos Aires: EUDEBA.

Manovich, L. (2013). Software Takes Command. New York: Bloomsbury Academic.

Metz, Ch. (1979). Metáfora/metonimia o el referente imaginario. En El significante imaginario-psicoanálisis y cine. Barcelona: Gustavo Gili.

Miller, D. et al. (2016). How the world changed social media. London: UCLPRESS Recuperado de www.ucl.ac.uk/ucl-press

Rudder, Ch. (2016). Dataclismo. Qué hacemos cuando creemos que nadie nos mira. E-book de Penguin-Random House: Grupo Editorial España.

Scolari, C.A.(2008). Delos nuevos medios alas hipermediaciones. En Hipermediaciones. Elementos para una teoría de la Comunicación Digital Interactiva (pp. 69-118). Barcelona: Gedisa.

Scolari, C. A. (2013) ¿Cómo se producen las narrativas transmedia? En Narrativas transmedia. Cuando todos los medios cuentan (pp. 61-78). Barcelona: Deusto.

Traversa, O. (2009). Dispositivo-enunciación: en torno a sus modos de articularse. En Figuraciones. Teorías y críticas de arte, 6. Recuperado de http://www. revistafiguraciones.com.ar/numeroactual/recorrido.php?idn=6\&idr $=48$.

Tusón Valls, A. (2002). El análisis de la conversación: entre la estructura y el sentido. En Estudios de Sociolingüística 3 (1), pp. 133-153.

Van Dijck, J. (2016). La cultura de la conectividad. Una historia crítica de las redessociales. Buenos Aires: Siglo XXI.

\section{IDENTIFICACIÓN DEL AUTOR}

José Luis Fernández es Doctor en Ciencias Sociales por la Facultad de Ciencias Sociales, Universidad de Buenos Aires (UBA). Premio a la Producción Científica y Tecnológica, UBA, 1994. Es profesor universitario y director de distintos proyectos de investigación con subsidio para investigadores formados, UBACyT, de la Secretaría de Ciencias y Técnica de la UBA desde 1995. Director de la Revista académica L.I.S. Letra, imagen, sonido. Ciudad mediatizada. Ha publicadolibrose innumerables artículos en revistas especializadas en temas de comunicación.

\section{REGISTRO BIBLIOGRÁFICO}

Fernández, J. L. (2016, diciembre). Plataformas mediáticas y niveles de análisis. En InMediaciones de la Comunicación, 11, pp. 71-96. Revista de la Escuela de Comunicación, Facultad de Comunicación y Diseño, Universidad ORT Uruguay, República Oriental del Uruguay. 